\title{
Wide-Dynamic-Range Forward Suppression in Marmoset Inferior Colliculus Neurons Is Generated Centrally and Accounts for Perceptual Masking
}

\author{
Paul C. Nelson, Zachary M. Smith, and Eric D. Young \\ Center for Hearing and Balance, Department of Biomedical Engineering, Johns Hopkins University, Baltimore, Maryland 21205
}

\begin{abstract}
An organism's ability to detect and discriminate sensory inputs depends on the recent stimulus history. For example, perceptual detection thresholds for a brief tone can be elevated by as much as $50 \mathrm{~dB}$ when following a masking stimulus. Previous work suggests that such forward masking is not a direct result of peripheral neural adaptation; the central pathway apparently modifies the representation in a way that further attenuates the input's response to short probe signals. Here, we show that much of this transformation is complete by the level of the inferior colliculus (IC). Single-neuron extracellular responses were recorded in the central nucleus of the awake marmoset IC. The threshold for a $20 \mathrm{~ms}$ probe tone presented at best frequency was determined for various masker-probe delays, over a range of masker sound pressure levels (SPLs) and frequencies. The most striking aspect of the data was the increased potency of forward maskers as their SPL was increased, despite the fact that the excitatory response to the masker was often saturating or nonmonotonic over the same range of levels. This led to probe thresholds at high masker levels that were almost always higher than those observed in the auditory nerve. Probe threshold shifts were not usually caused by a persistent excitatory response to the masker; instead we propose a wide-dynamicrange inhibitory mechanism locked to sound offset as an explanation for several key aspects of the data. These findings further delineate the role of subcortical auditory processing in the generation of a context-dependent representation of ongoing acoustic scenes.
\end{abstract}

Key words: inferior colliculus; marmoset; masking; context dependence; adaptation; suppression

\section{Introduction}

Sensory systems are exposed to a continuous barrage of complex stimuli, and our perception of specific components within such an environment is dependent on the temporal relationships between individual elements in the signal. A simple and robust example of temporal context dependence in hearing is forward masking, where the detectability of a short probe signal is degraded when a masking stimulus is presented before the probe. Forward masking contributes to changes in the neural representation of behaviorally relevant sounds such as consecutive speech phonemes, even in the auditory nerve (AN) (Delgutte, 1980).

Neural correlates of behavioral forward masking have been largely sought in AN responses (Relkin and Turner, 1988). However, the responses of single AN fibers (ANFs) do not account for two important aspects of psychophysical forward masking (see Fig. 1C). First, the masked threshold shift is proportional to the discharge rate during the masker in ANFs, so that neural masking has a limited dynamic range (often $<20 \mathrm{~dB}$ ). In contrast, psychophysical masking grows continuously over all suprathreshold lev-

Received Nov. 6, 2008; revised Jan. 14, 2009; accepted Jan. $28,2009$.

This work was supported by National Institutes of Health Grants DC00115, DC00023, and DC009164. We are grateful to Sean Slee, Bradford May, Sharba Bandyopadhyay, and two reviewers for comments on earlier versions of this work, and to Xiaogin Wang, Yi Zhou, Jenny Estes, and William Tam for help with animal preparation and care.

Correspondence should be addressed to Paul C. Nelson, 505 Traylor Research Building, 720 Rutland Avenue, Baltimore, MD 21205. E-mail: pcnelson@jhu.edu.

DOI:10.1523/JNEUROSCI.5359-08.2009

Copyright @ 2009 Society for Neuroscience $\quad$ 0270-6474/09/292553-10\$15.00/0 els (Lüscher and Zwislocki, 1949; Jesteadt et al., 1982; Plack and Oxenham, 1998). Second, maximum threshold shifts in AN responses $(<21 \mathrm{~dB})$ (Relkin and Turner, 1988) are much less than their psychophysical counterparts $(>40-50 \mathrm{~dB})$. As a result, the behavioral threshold is substantially higher than the peripheral single-neuron threshold at moderate and high sound pressure levels (SPLs). Higher processing centers seem to discard information present in the AN, possibly in an effort to maintain a robust representation of new or dynamic environmental signals.

Several questions surface when the psychophysics and the available physiology are considered together. Clearly, central mechanisms must shape the neural representation of these stimuli, but details of this transformation remain unclear. Can the psychophysical behavior be explained with responses of single neurons? If so, at what level of the pathway do the perceptually relevant response features emerge? How are forward-masked threshold functions related to simple tone-driven responses? And what is the mechanism of central forward masking? Is it better described as a persistence of the response to the masker (as assumed in many psychophysical models used to account for forward masking) (Moore et al., 1988), or as a decrease in excitability (i.e., adaptation or inhibition) following masker offset?

We measured forward-masked responses in single neurons in the central nucleus of the inferior colliculus (IC) of the awake marmoset. We found that responses of IC neurons could account for the behavior of growth of masking (GOM), in terms of wide dynamic ranges, maximum amounts of masking, and recovery 


\section{A. stimulus waveform}

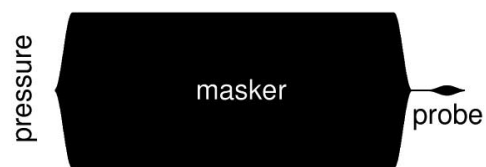

B. peripheral model response

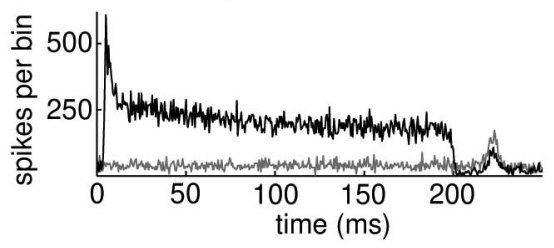

C. AN data (redrawn from Relkin and Turner, 1988)

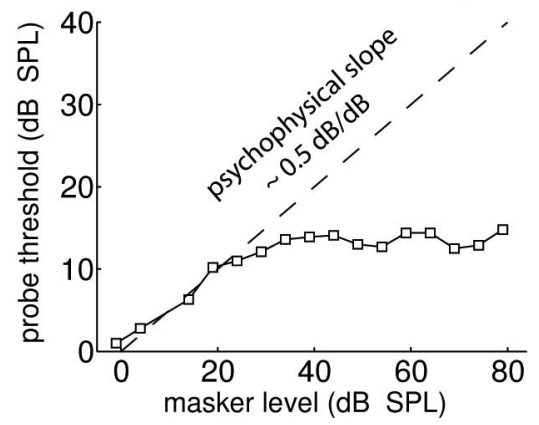

Figure 1. Stimulus paradigm and qualitative representation of the neural response in the AN. A, Sound pressure waveform of a $40 \mathrm{~dB} S P L, 4 \mathrm{kHz}, 200$ ms masker preceding a 15-dB SPL, $4 \mathrm{kHz}, 20 \mathrm{~ms}$ probe with a $10 \mathrm{~ms}$ delay between masker offset and probe onset. $B$, Simulated AN responses [Zilany and Bruce (2007) cat auditory nerve model] for probe-alone (gray) and masker-plus-probe (black) conditions. Model parameters: best frequency $=4 \mathrm{kHz}$; spontaneous rate $=50 \mathrm{sp} / \mathrm{s}$. A robust response to the probe is seen with or without the masker, although the response is smaller in the presence of the masker. $C, A N$ data from chinchilla showing growth of masking from the example in Figure 5 of Relkin and Turner (1988). The dashed line shows the approximate probe detection threshold for human observers with similar stimuli (Plack and Oxenham, 1998). In a population of ANFs, Relkin and Turner (1988) observed maximum threshold shifts that were almost always $<20 \mathrm{~dB}$ and sometimes as small as $3-5 \mathrm{~dB}$, even with no delay between the masker offset and probe onset [Relkin and Doucet (1991)]. In contrast, the maximum human psychophysical threshold shifts are larger, up to $30-50 \mathrm{~dB}$ (dashed line in $\mathbf{C}$.

from masking that was roughly linear in log time. The underlying mechanism of masking was almost universally adaptation or offset inhibition; $<10 \%$ of the population showed characteristics consistent with persistence of the masker response when best frequency $(\mathrm{BF})$ maskers were used. Our data provide the first examination of forward-masked thresholds in the central auditory system, and they suggest that neural mechanisms underlying the behavioral phenomena are largely established at the level of the IC.

\section{Materials and Methods}

Animal preparation. Animal care and recording procedures are similar to those used previously for studies in the awake marmoset auditory cortex and thalamus (Lu et al., 2001; Bartlett and Wang, 2007). During a onemonth period preceding surgery, the animal was adapted to sitting quietly in the restraint device for the $2-4 \mathrm{~h}$ period used for recording. A sterile, isoflurane-anesthetized surgery was performed to mount posts for subsequent head fixation during recordings and to expose a small portion of the skull to provide access to the IC. The head posts were surrounded by dental acrylic, which bonded to the skull and also to a set of stainless steel screws that were mounted in the skull.

After a 2- to 3-week recovery period including daily antibiotic treatment and wound cleaning and maintenance, a small ( $\sim 1$-mm-diameter $)$ craniotomy was made in the skull. The location of the hole was based on stereotaxic coordinate maps as well as superficial landmarks on the skull that were marked during the sterile surgery (e.g., the lateral sulcus). The exposed recording chamber surrounding the craniotomy was covered with polysiloxane impression material (GC America, Alsip, IL) between recording sessions, and after many penetrations (usually $>20$ ), the hole was filled with a layer of bone wax and dental acrylic before making another craniotomy to provide access to other regions of the same IC. Multiple craniotomies were performed in both hemispheres before the animal was killed and perfused for histological evaluation. All procedures were approved by the Johns Hopkins University Institutional Animal Care and Use Committee and conformed to National Institutes of Health standards.

Recording methods. Once the craniotomy was made, an epoxy-coated tungsten microelectrode (A-M Systems) was slowly advanced during daily recording sessions using a hydraulic microdrive (Kopf Instruments). During recording, the animal was awake or drowsing with its body loosely restrained in a comfortable seated position with the head held in a natural orientation. Depending on the angle of approach (which varied across animals and sessions), the electrode traversed $0.8-1 \mathrm{~cm}$ of brain tissue before reaching the IC. Usually the electrode was in an approximately frontal plane at an angle of $\sim 45^{\circ}$ lateral to medial. Stimulus presentation, animal monitoring via video camera, and electrode advancement were controlled from outside a double-walled soundproof booth. Spike times of Schmidt-triggered single-neuron responses were recorded with respect to stimulus onset. Only well isolated single neurons were studied. Sessions were terminated after 3-4 h, or earlier if the animal showed signs of discomfort. Using these techniques, it was possible to collect data from several hundred electrode penetrations over the course of more than a year per animal.

Often, the external nucleus (equivalently, lateral cortex) (Oliver, 2004) or dorsal cortex of the IC was encountered using our lateral-access approach. We assumed that neurons were located in the CNIC based on three criteria: (1) short-latency (minimum first-spike latencies less than $\sim 20 \mathrm{~ms}$ ), (2) reliable and qualitatively nonhabituating responses, and (3) a well defined location within the tonotopically organized frequency lamina (Aitkin et al., 1975). Neurons meeting these criteria are called "CNIC-like"; data from neurons not meeting these criteria are not included in the results presented here.

Acoustic stimuli. Sound stimuli were digitally generated in Matlab (Mathworks), converted to analog signals (RP2.1, Tucker-Davis Technologies) and presented in the free field through a speaker (FT-28D, Fostex) positioned $\sim 1 \mathrm{~m}$ directly in front of the animal. With few exceptions, previous studies have tended to use monaural earphone stimulation in nuclei below the IC (Relkin and Turner, 1988; Shore, 1995), binaural headphone stimulation in IC (Sanes et al., 1998; Finlayson, 1999), and free-field stimuli at higher levels of the pathway (Bartlett and Wang, 2005; Scholl et al., 2008). No systematic differences in forward masking related to the stimulation mode are apparent across physiological or psychophysical studies. The free-field stimuli contained reverberation of the stimuli produced by the speaker, despite the foam padding on the chamber walls. The reverberation lasted $\sim 10 \mathrm{~ms}$ but was at a low level $(<20 \mathrm{~dB})$ relative to the stimuli. The strongest potential effect of such reverberation would be to produce a persistence of the response to the masker stimulus. As we show, such persistence was seen very rarely in the responses of neurons, suggesting that the impact of the weak acoustic echoes on the data was small or negligible.

Wideband noise bursts (50 or $200 \mathrm{~ms}$ in duration, $48.8 \mathrm{kHz}$ bandwidth) and tone bursts of variable frequency and level were used to characterize neural activity as sound-driven and to estimate BF and threshold. BF was defined as the frequency at which sound-driven responses were observed at the lowest SPL. Each neuron was characterized by its response map (RM) (Ramachandran et al., 1999) and rate-level function (RLF). To obtain a RM, $200 \mathrm{~ms}$ tones (10 ms rise/fall) were presented (1/s) over a $2-4$-octave range centered on the previously estimated BF, using 25 log-spaced frequencies per octave. This procedure was repeated for at least three sound levels, usually 20,40 , and $60 \mathrm{~dB}$ above the audio-visually determined estimate of BF threshold. RLFs were measured with $200 \mathrm{~ms}$ stimuli over a range from approximately $-10-85$ 


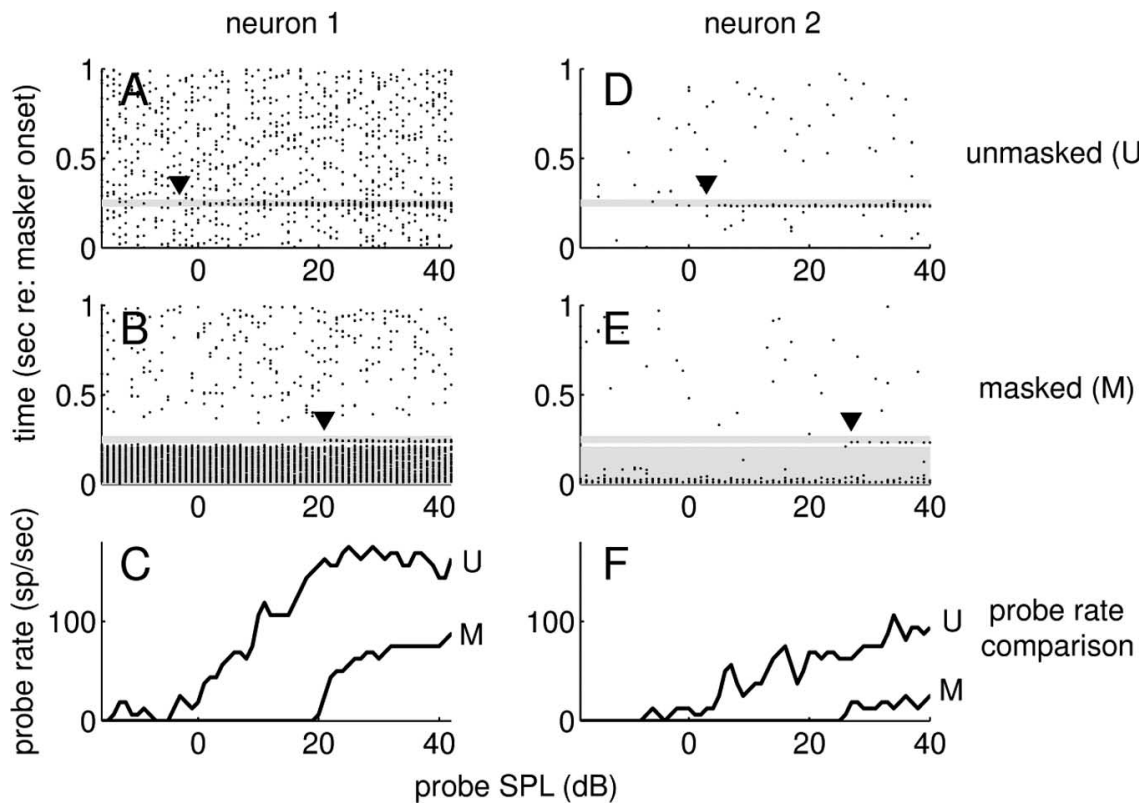

Figure 2. Example of masked and unmasked responses for two neurons ( $40 \mathrm{dBSL}, 200 \mathrm{~ms}$ BF-tone masker with a $10 \mathrm{~ms}$ probe delay). For each probe $S P L$ value along the abscissa, spike-time rasters are shown with time increasing on the ordinate $(\boldsymbol{A}, \boldsymbol{B}, \boldsymbol{D}$, and $\boldsymbol{E})$. Gray areas show the masker timing and probe response analysis window. Downward arrows are positioned at the threshold probe sound level. $\boldsymbol{C}, \boldsymbol{F}$, Rate functions computed in a $40 \mathrm{~ms}$ window surrounding the probe response for the two stimulus conditions. BF, spontaneous rate, neuron type, unmasked threshold, and masked threshold are $4.1 \mathrm{kHz}, 17.5 \mathrm{sp} / \mathrm{s}$, type I, $-3 \mathrm{~dB}$, and $21 \mathrm{~dB}$ for neuron 1 , and $14.2 \mathrm{kHz}, 1.4 \mathrm{sp} / \mathrm{s}$, onset, $3 \mathrm{~dB}$, and $27 \mathrm{~dB}$ for neuron 2.

A

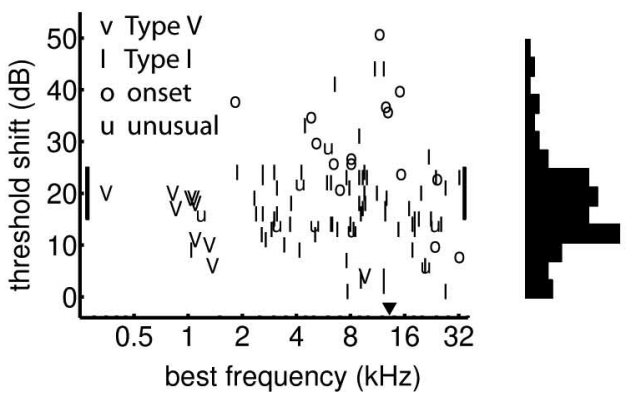

B

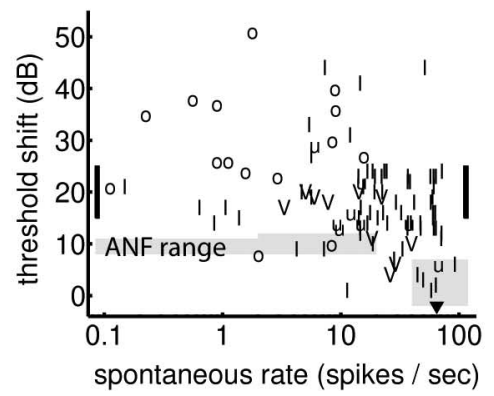

Figure 3. Amount of masking across the population in default masker conditions. $\boldsymbol{A}$, Threshold shift versus neuron BF. Different symbols correspond to different unit types (type I, type $V$, onset, and unusual). Type 0 neurons were not included. $\boldsymbol{B}$, Threshold shifts as a function of the neurons' spontaneous firing rates. Vertical bars framing the plots between 15 and $25 \mathrm{~dB}$ mark the approximate range of threshold shifts measured under similar conditions in psychophysical forward masking. Gray-shaded areas show the range of threshold shifts observed in chinchilla AN fibers under nearly identical stimulus conditions [Relkin and Doucet (1991)]. Filled downward triangles represent one type I unit with $-27 \mathrm{~dB}$ of masking. The histogram between $\boldsymbol{A}$ and $\boldsymbol{B}$ collapses threshold shifts across BF and spontaneous rate; its peak corresponds to 21 neurons.

Table 1. Average threshold shifts and SDs for each class of neurons grouped by frequency response map type for the default stimulus condition

\begin{tabular}{lccc}
\hline & Mean & $\sigma$ & $N$ \\
\hline Type I & 17.6 & 8.6 & 68 \\
Type V & 14.4 & 6.1 & 10 \\
Onset & 28.8 & 11.3 & 15 \\
Unusual & 16.0 & 6.8 & 8 \\
\hline
\end{tabular}

The average shift for onset neurons was greater than that in any of the other categories (see text).

dB SPL (with SPL defined as the sound level at the position of the animal's head in the absence of the animal) in $1 \mathrm{~dB}$ steps, using both BF tones and broadband noise bursts. Spontaneous rate was estimated using the $400 \mathrm{~ms}$ periods immediately preceding sound onset, and was used as another part of the basic characterization of the neurons. All stimuli were presented once; when plotted, rate functions were smoothed using a triangular window spanning three consecutive SPLs.

With the more precise values of $\mathrm{BF}$ and threshold obtained from the RM and BF RLF, a family of forward-masked RLFs was measured. First, a control RLF was obtained using a $20 \mathrm{~ms}$ $\mathrm{BF}$ probe alone $(10 \mathrm{~ms}$ rise/fall, no steady state, repetition rate of $1 / \mathrm{s})$, over a range of $50-70 \mathrm{~dB}$. The lowest tested probe SPL was at least $10 \mathrm{~dB}$ below the threshold estimated from the $200 \mathrm{~ms}$ tone RLF. Following this baseline condition, forward-masked RLFs were measured, consisting of a $200 \mathrm{~ms}$ masking tone followed by the probe tone (as in Fig. 1A). The masker had fixed properties and the probe level was varied as above. The default parameter values for the masker were a frequency at BF, an SPL $\sim 40 \mathrm{~dB}$ above the neuron's threshold, and a delay between the masker offset and probe onset of 10 $\mathrm{ms}$. The forward-masked paradigm was repeated with various combinations of two of these parameters fixed and the third varied, until contact with the neuron was lost. Masker SPL was varied in 10-20-dB steps from near threshold levels to 60-80 dB SPL above threshold. Masker-offset/probe-onset delay was varied from $0 \mathrm{~ms}$ up to $310 \mathrm{~ms}(0,10,30,70,150$, and $310 \mathrm{~ms})$. Finally, the frequency of the masker tone was varied in some neurons. The frequencies chosen were based on features of the puretone responses, often within inhibitory regions of the RM.

Response analysis. For direct comparison with psychophysical data, we focused on describing the changes in probe detection thresholds given different parameter variations in the masker. Threshold was defined as the lowest probe level that elicited a reliable increase in response rate in a $40 \mathrm{~ms}$ window surrounding the probe. The beginning of the window was delayed by $10 \mathrm{~ms}$ following the physical probe onset to allow for acoustical and neural delays. Probe threshold was determined based on a comparison response which was defined as the average rate observed for the 10 stimulus conditions at the lowest probe levels (all set to SPLs below the threshold for $200 \mathrm{~ms}$ tones). For each probe SPL above the lowest 10 tested, a "hit" was recorded if the number of spikes in the test window was greater than the comparison spike count. Otherwise, the trial was labeled a "miss." Probe threshold was empirically defined as the lowest level in a string of 10 or more probe SPLs that resulted in a hit, with no more than a single miss allowed between otherwise consecutive hits.

This threshold criterion was adopted because of our decision to use high resolution when varying probe SPL (1 dB steps), as opposed to collecting multiple repetitions of each stimulus condition. This is in contrast to the method used by Relkin and colleagues (Relkin and Pelli, 1987; Relkin and Turner, 1988), who collected many repetitions of a more sparsely sampled probe SPL space. Our definition was almost always consistent with the threshold determined by directly examining dot rasters, and was actually trivial in many conditions where the comparison spike rate was zero. If anything, our rate-based rule would tend to underestimate sensitivity in probe-alone conditions because of the higher background comparison rate (as in ANF responses). The reliability of our method was supported by the reliability of threshold estimates in simu- 
lations of Poisson spike-trains with rates typical of our data and smoothing to produce comparable variability in the spike counts.

Excluded neurons. Type O neurons (Ramachandran et al., 1999) were not common in this population, but four that were encountered were excluded from the analysis because they gave weak and nonmonotonic probe responses that were usually completely suppressed by the presence of a preceding masker (i.e., masked thresholds were undefined). The responses of a single type $V$ neuron were not included for similar reasons: the probe response did not reach the criterion for threshold over the entire range of levels tested. Neurons were included in the population analyzed for different probe delays only if thresholds were measured for at least three masker-probe delays with a masker presented at an SPL $\sim 40 \mathrm{~dB}$ above threshold. One additional neuron was excluded because thresholds did not recover toward probe-alone conditions, even after a $320 \mathrm{~ms}$ masker-probe delay (the probe threshold shift remained larger than $40 \mathrm{~dB}$ for all tested delays); the interstimulus delay was apparently not long enough in this case to avoid cumulative adaptation.

\section{Results}

IC responses to the default masker

The responses to the forward-masking paradigm were recorded from 102 CNIClike neurons from two animals; recordings were made in the IC on both sides. When the masker and probe tone frequencies were both set to the neuron's BF, the effect of the masker on the probe response was almost exclusively suppressive over the entire range of probe levels tested (usually $\geq 50 \mathrm{~dB}$ ).

The responses of two example neurons to a $20 \mathrm{~ms}$ BF probe tone over a range of probe SPLs are illustrated in Figure 2, in the form of spike-time raster plots (Fig. $2 A, B, D$, and E) and ratelevel functions (Fig. 2C,F). Probe-alone (unmasked) responses are shown in Figure 2, $A$ and $D$, and responses to the sequential presentation of the default masker $[40 \mathrm{~dB}$ sensation level (SL) BF tone] and the subsequent probe are shown in Figure, 2, $B$ and $E$. Downward triangles indicate the neural detection threshold for the probe; shaded areas mark the timing of the masker (when present) and the $40 \mathrm{~ms}$ probe analysis window. For each of these neurons (and for 101 of 102 of the population), the preceding masker caused an increase in the neural detection threshold for the probe. This can be seen as a rightward shift in the triangles in the second versus the first row of panels. The two examples include a neuron with sustained responses to the masker (Fig. $2 \mathrm{~A}-$ $C$ ), as well as a transient (onset) responder to both the masker and the probe (Fig. 2D-F). Both neurons exhibited a forwardmasked threshold shift of $24 \mathrm{~dB}$.

Across the population, the average amount of masking was $18.8 \pm 9.6$ (mean and SD) $\mathrm{dB}$ for the default masker. This value is well within the range of shifts observed psychophysically across studies and subjects for comparable stimulus conditions ( 15-25 dB) (Jesteadt et al., 1982; Plack and Oxenham, 1998). The amount of masking was not strongly dependent on BF, although the widest range of threshold shifts was found in the midfrequency $(4-16 \mathrm{kHz})$ region, and we did not observe any shifts larger than $20 \mathrm{~dB}$ in neurons with BFs of $<1.5 \mathrm{kHz}$ (Fig. $3 A$ ). These low-frequency neurons tended to have $\mathrm{V}$-shaped frequency response maps, with a pronounced lack of sideband inhibition above or below BF.

In contrast to the near independence of threshold shift and $\mathrm{BF}$, there was a weak but significant dependence of the amount of masking on spontaneous rate (SR): low-SR neurons tended to exhibit more masking than high-SR neurons (Fig. 3B) [amount of masking vs $\log (\mathrm{SR})$ correlation, $r=-0.35 ; p<0.001]$. This is also true of ANFs (Relkin and Doucet, 1991), but the magnitude of the threshold shift in the IC population was much greater than that observed in ANFs. The gray hatched areas in Figure $3 B$ represent the range of ANF threshold shifts measured under nearly identical stimulus conditions [redrawn from the work of Relkin and Doucet (1991), their Fig. 3]. The largest threshold shifts were observed in onset neurons (Table 1$)(2 \times 2$ contingency test, $p<$ 0.06 for all combinations of onset and other unit types) with no significant difference between the other neuron types $(2 \times 2$ contingency test $p>0.17$ for the remaining combinations).

\section{Response dependence on masker level}

As discussed above, in ANFs forward masking varies with the discharge rate in response to the masker, typically leading to saturating GOM curves (Fig. 1C) (Relkin and Turner, 1988). IC neurons show a variety of RLFs, many showing nonmonotonic behaviors. However, GOM in IC neurons with the forward masking paradigm usually did not exhibit similar nonmonotonicities. 

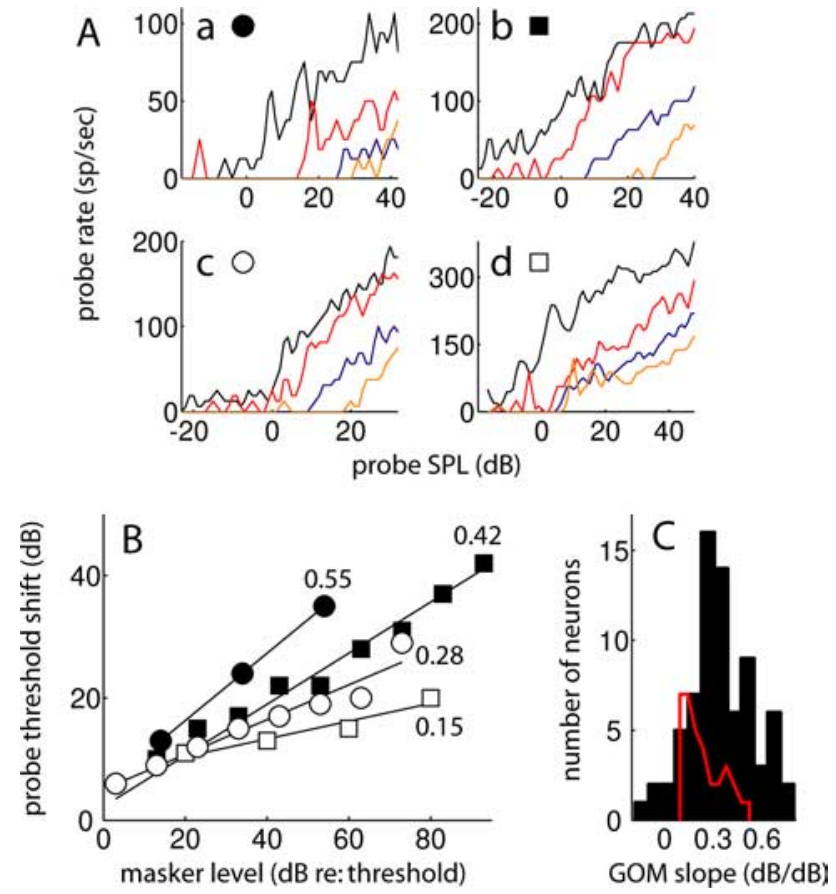

Figure 5. Probe threshold increases monotonically and linearly as masker level increases. $\boldsymbol{A}$, Probe rate functions for four IC neurons. Black lines are for probe-alone conditions; other lines correspond to different masker SPLs. Masker levels used (dB SL) for each neuron (probe responses drawn in red, blue, and orange, respectively) are as follows: $\boldsymbol{a}:[25,45,65] ; \boldsymbol{b}$ : $[15,55$, 95]; c: [10, 40, 70]; $\boldsymbol{d}$ : [40, 60, 80]. $\boldsymbol{B}$, GOM functions for the four neurons shown in $\boldsymbol{A}$. Solid lines are fit to each set of points to estimate the slope of the GOM function (slopes labeled next to each line). C, GOM slopes for IC neurons in this study (black histogram) and for AN fibers [red line; extracted from the work of Relkin and Turner (1988), their Fig. 7]. AN slopes were calculated from a limited (low-masker-level) portion of the GOM functions, while IC slopes were derived from the entire range of masker levels tested (a range as large as $90 \mathrm{~dB}$ ). One IC neuron is not shown, with a slope of $1.46 \mathrm{~dB} / \mathrm{dB}$.

We found that most (51 of 74) IC neural GOM functions increased monotonically over the $40-80 \mathrm{~dB}$ range of masker levels tested, despite saturation or turn-over in the excitatory maskerdriven RLFs ( 49 of 74 had saturating or nonmonotonic RLFs over the same SPL range).

Spike-time rasters for three neurons' responses to variations in masker SPL (across different panels) and probe SPL (within panels) are shown in Figure 4. When presented in isolation, the short probes reliably evoked a spike or burst of spikes at a relatively low probe SPLs; detection thresholds are shown by the downward triangles as before. These examples illustrate the difference between the behavior of the probe threshold and the rate in response to the masker (given by the numbers to the right of the dot displays). In the three examples shown, detection threshold shifted monotonically with masker level in the face of a nonmonotonic masker response (left column), essentially no sustained response to the masker (middle column), or a saturating response to the masker (right column).

Typical probe rate functions are shown in the panels of Figure $5 A$ for four other example neurons. The parameter in each panel is masker SPL, and different colors are used for different SPLs of the preceding masker (masker responses not shown). These examples show a suppression of the probe response magnitude over the entire range of probe SPLs tested, with the strongest suppression and largest threshold shift caused by the loudest masker. Thresholds derived from these probe responses were used to construct individual-neuron GOM functions (Fig. 5B).
A straight line was fit to each set of points in Figure $5 B$ to determine the GOM slope. The slopes in these example neurons were $0.55,0.42,0.28$, and 0.15 (Fig. $5 A a$ - $d$, respectively). Across the population, the average GOM slope was 0.36 . A comparison of GOM slopes between IC neurons (Fig. 5C, shaded histogram) and ANFs [Fig. 5C, red line redrawn from Relkin and Turner (1988)] reveals a tendency for IC GOM slopes to be greater than those measured in the AN (Wilcoxon rank-sum test $p<0.02$ ). This is true although the AN slopes were computed only for masker levels on the dynamic portion of the ANF's RLF (typically 20-30 dB SL) and used no gap between the masker and probe; the IC slopes were derived from probe responses over the entire range of masker levels tested (i.e., including levels that potentially caused a saturated or decreased high-level response to the masker) and a $10 \mathrm{~ms}$ masker-probe gap.

On average, the amount of masking in IC neurons grew gradually as a function of masker level (Fig. 6A), with no sign of hard saturation at the highest masker levels tested. The dashed line in Figure $6 \mathrm{~A}$ has a slope of $0.5 \mathrm{~dB} / \mathrm{dB}$ and assumes no threshold shift for a masker presented at $0 \mathrm{~dB}$ sensation level.

\section{On-BF masking is not consistent with persistence}

Inspection of the dot displays in Figures 2 and 4 shows that there is a clear gap between the response to the masker and the response to the probe. This behavior shows that the persistence model often used in psychophysical studies (Moore et al., 1988; Oxenham, 2001) does not apply to the IC; in that model, masking occurs because of persistence of response to the masker, which overlaps with the response to the probe. To show that this behavior was typical, we used a persistence/adaptation ratio (PA ratio: the average rate in the probe analysis window in the masker-alone condition divided by the average spontaneous rate). Values less than one indicate an adaptation or inhibition-driven mechanism; values greater than one suggest a persistent or offset excitatory response. Individual points contributing to the averaged GOM function are shown in Figure $6 B$, with the symbol size related to the value of the PA ratio. Clearly, a persistent response was not required to generate a threshold shift comparable with those observed psychophysically (small points correspond to small PA ratios).

The relatively few persistent (or offset) responses that we observed did not emerge until the masker level exceeded $\sim 30 \mathrm{~dB}$ SL. The vast majority of masker conditions resulted in PA ratios of $<1$ (259 of $312,83 \%$ ), and more than half ( 170 of 312 ) elicited no spikes in the masker-alone observation window $(\mathrm{PA}$ ratio $=0$ ) (Fig. 6D). Consistent with the independence of individualneuron GOM and masker RLF slopes (above), there was not a strong relationship between threshold shift and the maskerdriven rate when the entire population was considered (Fig. 6C).

\section{Response dependence on masker-probe delay}

Psychophysical forward masking (in $\mathrm{dB}$ ) is proportional to the log of signal delay (Lüscher and Zwislocki, 1949; Widin and Viemeister, 1979; Kidd and Feth, 1982; Jesteadt et al., 1982; Moore and Glasberg, 1983). In other words, probe threshold shifts exhibit a linear dependence on signal delay when delay is plotted on a logarithmic scale. Here we show a similar behavior for IC neurons.

In a subset of neurons $(n=41)$, we examined the effect of changing the delay between masker offset and probe onset, over a range from 0 to $310 \mathrm{~ms}$. Responses of two example neurons are shown in Figure 7A. Spike-time raster plots are overlaid in different colors for the different delay conditions, although the condi- 
tions were presented in separate independent blocks. The 40-dB SL, 200 ms masker elicited a sustained response in the first neuron, and a transient onset response in the other cell. Responses to the probe emerged at higher SPLs for probe signals presented with shorter delays. For these examples, the probe threshold decreased with delay and recovered toward baseline (unmasked) conditions. Responses to the probe-alone case (Fig. $7 A$, right column) emerged at lower probe sound levels than the masked responses, and were also larger in magnitude at suprathreshold levels.

Probe threshold recovery functions for individual neurons are shown in Figure $7 B$ (thin lines), along with the across-neuron average (thick line with circles, $\pm \mathrm{SD}$ ). It is clear that most of the probe thresholdshift functions, along with the average curve, do recover approximately linearly in log time (even ignoring the point at 0 delay which is arbitrarily positioned on the $\log$ abscissa). There is a small amount $(\sim 5$ $\mathrm{dB})$ of residual masking that does not change between the 150 and 310 ms delay conditions. This was observed despite the fact that the silent time between the probe offset and masker onset was always $>500$ $\mathrm{ms}$, an off-period that is typical in psychophysical measurements. The magnitude of this residual masking was likely amplified by a bias in the data collection: neurons that showed little or no masking for shorter delays were often not tested at the longest delay.

\section{Response dependence on masker frequency}

Generally, maskers had their largest effect when placed at BF. As has been shown above, the type of masker-driven response pattern (i.e., sustained or onset) was secondary. To illustrate this behavior, the responses of three neurons are shown in Figure 8 for stimuli with various masker frequencies. The neuron in Figure $8 \mathrm{~A}$ was studied with maskers at three frequencies, all within the excitatory region of the response map. Each masker produced a qualitatively similar sustained spiking pattern throughout the masker (driven rates during the masker were 30, 80, and $57 \mathrm{sp} / \mathrm{s}$, for the below-BF, on-BF, and above-BF maskers, respectively). The on-BF masker had the largest forward masking effect (blue circle), producing a $17 \mathrm{~dB}$ shift in probe threshold and a large decrement in the rate response to the probe (Fig. $8 A b$ ). The other two masker frequencies produced much smaller threshold shifts (filled square and diamond).

Usually, maskers positioned within an inhibitory region of the frequency response map did not cause significant elevations in probe threshold (Fig. $8 \mathrm{Bb}$, orange square and red diamond). In fact, maskers in the inhibitory regions of the response map could produce a modest improvement in probe thresholds over the unmasked condition (11 of 39 tested). This "negative masking" had a magnitude between 5 and $12 \mathrm{~dB}$, with one unusually high spontaneous-rate neuron exhibiting negative masking $>20 \mathrm{~dB}$ for all masker conditions tested (this neuron was the outlier omitted from the population plots in Fig. 2).
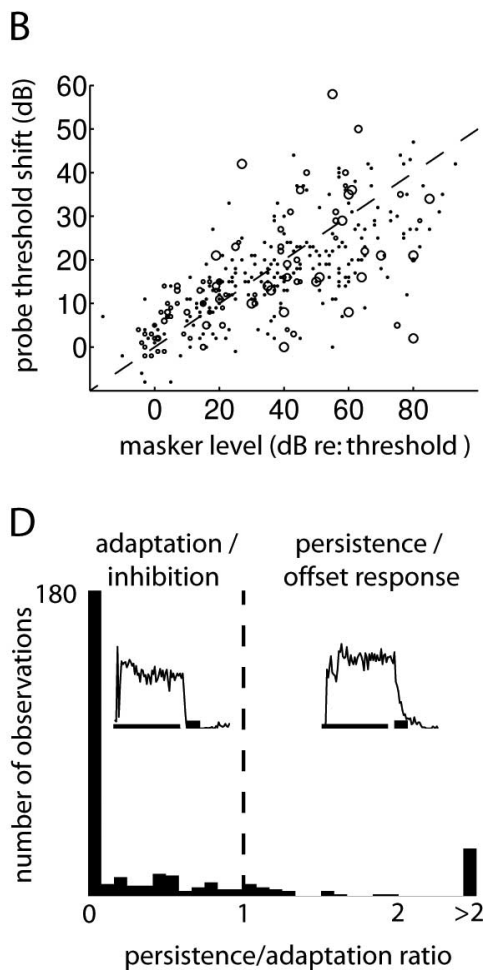

Figure 6. Growth of masking (GOM) across the population. $A$, Across-neuron ( $n=74)$ average probe threshold shift ( \pm SD) versus masker level. The dashed line represents the psychophysical G0M slope of $0.5 \mathrm{~dB} / \mathrm{dB}$. $\boldsymbol{B}$, Individual points contributing to $\boldsymbol{A}$. Each neuron is represented between three and eight times (mean $=4.2$ points/neuron). The size of the symbol corresponds to the (PA) ratio for each condition, with the largest symbols representing PA ratios of $>4$ and the smallest side of the PA ratio = 1 dashed line; the timing of the masker and probe (when presented) are indicated beneath the PST histograms. One neuron responded with zero spontaneous spikes in one or more conditions; its PA ratio was arbitrarily set to zero.

In some cases, the response to the probe was affected by offset discharges in response to off-BF maskers. The example in Figure $8 C$ shows the most extreme example of such a case: the above-BF masker (red diamonds) caused such a strong offset response (compare red and black RLFs at low probe SPLs) that the addition of the probe did not cause a further increase in firing rate over the entire range of probe levels tested. This neuron is identified in Figure 9 (see below) by symbols surrounded by parentheses to indicate its status as an outlier in this sense.

The data in Figure 8 are typical in that the largest masking was observed within the excitatory response area of the neuron; that is, the masking was often tuned at least as narrowly as the excitatory response of the neuron. Figure 9 shows population data on this point. On-BF maskers (blue symbols) generally produced more masking (ordinate) than off-BF maskers (red symbols). Again, the amount of masking was not predicted by the discharge rate during the masker (Fig. 9A). However, some inhibitory maskers (negative masker-driven rates) produced as much masking as on-BF maskers. Those were mostly cases with PA ratios of $>1$ (Fig. 9A, open symbols), in which the masking was produced by an offset response as in Figure $8 C$. The decline of forward masking for this group of neurons as the masker frequency is moved away from BF is shown in Figure $9 B$.

\section{Discussion}

The nature of physiological forward masking in IC

To our knowledge, these data represent the first description of neural forward-masked detection thresholds at any level of the 

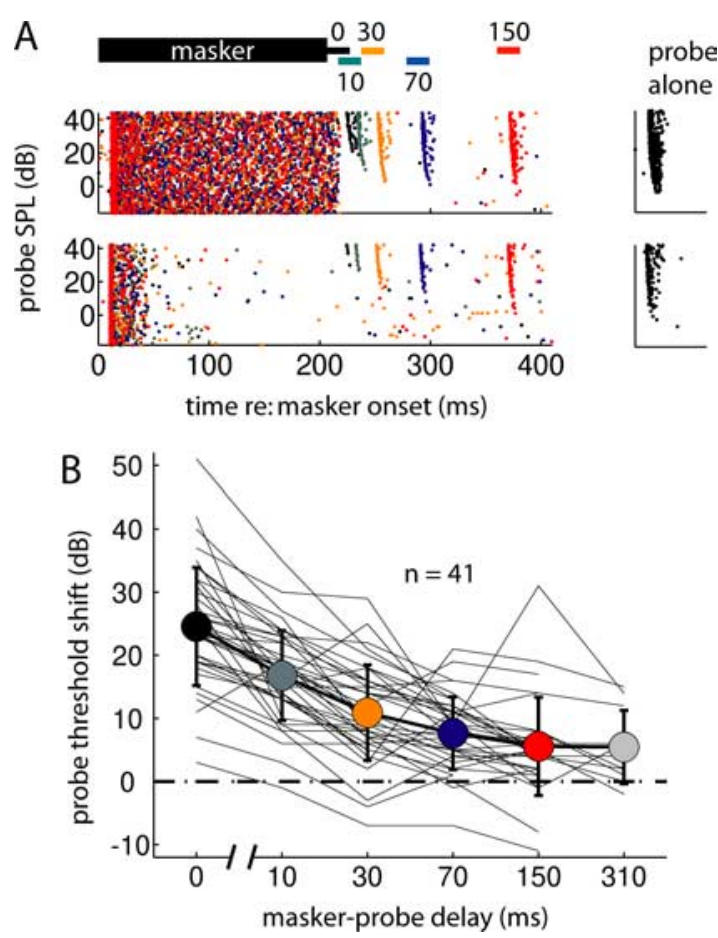

Figure 7. Probe response delay dependence. $\boldsymbol{A}$, Two individual neurons' masked and unmasked (probe alone) spiking responses to masker-probe sequences separated by a range of delays $(0-150 \mathrm{~ms})$. Spike times in the panels including the masker response are overlaid for all five delay conditions (resulting in masker responses that appear denser than they would on a single trial). The dots for each delay condition are shown in different colors, identified by the labels at the top. Each delay was actually presented separately. The probe-alone response window shown at right is $65 \mathrm{~ms}$ long. $\boldsymbol{B}$, Amount of forward masking in each of the 41 neurons tested with different delays using a BF masker presented $40 \mathrm{~dB}$ above threshold. The acrossneuron average (circles \pm SD) probe threshold shift is also shown.

central auditory system. A large number of papers have described forward masking at various levels of the auditory system [AN: Smith (1977), Harris and Dallos (1979); CN: Starr (1965), Boettcher et al. (1990), Palombi et al. (1994), Shore (1995), Backoff et al. (1997), Bleeck et al. (2006); SOC: Finlayson and Adam (1997); IC: Finlayson (1999), Faure et al. (2003), Furukawa et al. (2005); A1: Calford and Semple (1995), Brosch and Schreiner (1997), Brosch et al. (1999), Bartlett and Wang (2005), Wehr and Zador (2005), Nakamoto et al. (2006), Scholl et al. (2008)]. However, these have all considered the effect of the masker on the responses to a fixed probe stimulus. Because of nonlinear relationships between neural responses and detection threshold, it is not possible to estimate detection thresholds from these data and therefore not possible to compare them directly to behavioral data. Thus we considered it important to explicitly estimate probe thresholds.

The principal result of this study is that GOM in the awake marmoset IC resembles the monotonic $0.5 \mathrm{~dB} / \mathrm{dB}$ growth seen in human psychophysical forward masking (Plack and Oxenham, 1998) and not the saturating growth seen in anesthetized chinchilla ANFs (Relkin and Turner, 1988). Probe masking in the IC also shows a linear decay with log time (Fig. 7) and shows stronger masking for on-BF maskers (Figs. 8, 9), both features seen in psychophysical forward masking, although the frequency dependence cannot be quantitatively compared with the data available. Although there are no forward masking psychophysical data available for marmosets, we would expect that at least the gross trends in the fundamental perceptual features of the phenome- non would be conserved across primate species. An additional implicit assumption underlying our interpretation is that AN responses in the chinchilla are similar to those in primates.

The results also clearly show that masking does not depend on the net masker-driven excitatory activity in the cell (Figs. 6C, 9). Other studies examining the temporal context dependence of responses in the mammalian IC have reached conflicting conclusions as to whether the history of the excitatory spiking response is sufficient to account for the observed "adaptation" effects (McAlpine et al., 2000; Ingham et al., 2001) or not (Malone and Semple, 2001). The current data support the idea that the history of excitation is insufficient to predict the magnitude of forward masking in the marmoset IC. Another way of stating this conclusion is that IC forward masking is not strictly an adaptation process defined as a change in a neuron's properties due to its history of activation.

\section{Potential inhibitory mechanisms}

Inspection of the probe RLFs (Figs. 2, 4, 5) show a universal decrease in overall probe response rates relative to the unmasked condition, including a suppression of spontaneous activity. This behavior suggests that inhibitory mechanisms are involved in forward masking in the IC, either within the IC or at lower levels of the auditory system. There is considerable evidence for poststimulus inhibitory activity in IC. Sound-evoked IPSPs and currents are present in IC neurons following the cessation of the acoustic stimulus (Nelson and Erulkar, 1963; Kuwada et al., 1980, 1997; Covey et al., 1996; Pedemonte et al., 1997; Tan and Borst, 2007; Xie et al., 2008). Also, local blockade of inhibitory neurotransmitters $\left(\mathrm{GABA}_{\mathrm{A}}\right.$, and to a lesser extent, glycine) releases extracellular responses from the persistent (i.e., continuing beyond stimulus offset) inhibition that is often present in control conditions (Faingold et al., 1991; Pollak and Park, 1993; Bauer et al., 2000; LeBeau et al., 2001).

A parsimonious explanation for several aspects of the data relies on inhibitory inputs from the superior paraolivary nucleus (SPON) to generate the strong and wide-dynamic-range offset suppression of the probe. Many SPON neurons make a direct inhibitory (GABAergic) projection to the ipsilateral IC (Kelly et al., 1998; Kulesza and Berrebi, 2000), and their physiological responses are often characterized by no response during the stimulus and a burst of spikes locked to the offset of a tone burst that can last as long as $50 \mathrm{~ms}$ (Kuwada and Batra, 1999; Kulesza et al., 2003). These offset responses (1) tend to have higher thresholds than nearby "primary-like" SOC neurons, (2) are sharply frequency tuned near threshold and become more broadly tuned at higher SPLs, and (3) can exhibit rate responses with remarkably wide dynamic ranges (Kuwada and Batra, 1999; Kulesza et al., 2003). Such input and response patterns could at least qualitatively account for enhanced suppressive strength in the IC at higher masker SPLs (Figs. 2-5) and sharp tuning of this suppression (Figs. 8, 9). A schematic illustration of the frequencyresponse profile of a hypothetical IC neuron receiving such an input is shown in Figure 10. At low SPLs, the response to the masker is sharply tuned and purely excitatory, and any forward suppression is presumably inherited from lower levels. At higher SPLs, broadly tuned inhibitory inputs (possibly from the DNLL) are activated and interact with the excitation (Ramachandran et al., 1999). This inhibition could be sluggish, working to augment the forward suppression found in the inputs by lasting beyond the offset of stimulation. Finally, the SPON-IC pathway is active at still higher SPLs, potentially providing the substrate for monotonically increasing GOM functions in IC neurons. 
Other possible sources of forward suppression include intrinsic membrane properties in IC (e.g., GABA receptors with slow kinetics or a voltage-sensitive current that changes its properties following masker stimulation), local recurrent inhibition within the IC, and descending influences from auditory cortex. Interestingly, forward suppression in auditory cortex is not related to the strength of poststimulus hyperpolarization (Scholl et al., 2008), and patterns of probe suppression are broadly similar to those we recorded extracellularly in IC neurons. This, along with the tight match between behavior and IC responses we report, suggests that much of the processing underlying behavioral forward masking is set up before the thalamocortical pathway.

\section{Functional implications}

The so-called temporal-window model has influenced much of the recent interpretation of nonsimultaneous perceptual masking (Moore et al., 1988; Plack and Oxenham, 1998; Oxenham and Plack, 2000). Forward masking in this model results because the masker response is assumed to last beyond the stimulus offset in the form of persistent excitation, and responses to the subsequent probe tone are swamped by the extended masker-induced firing. While we did occasionally find persistent excitation in a small group of neurons at high masker levels (Fig. 6B,D), poststimulus inhibition was much more common. Importantly, neural threshold shifts consistent with psychophysical masking were regularly observed in the IC over the entire range of tested masker SPLs even if the few persistent neurons were excluded (Fig. $6 \mathrm{~B}$ ). We conclude that mechanisms like inhibition or adaptation (discussed above) are more likely to account for behavioral forward masking, despite circumstantial psychophysical evidence suggesting otherwise (Oxenham, 2001) and the relatively weak offset adaptation that is present in ANFs (Relkin and Turner, 1988).

Differences in the susceptibility of different neuron types to conditioner-induced suppression (Table 1, Fig. 3) may provide a hint as to their functional importance in hearing. Some IC neurons show small threshold shifts (similar to those measured in ANFs); this information is apparently unavailable or not used by listeners in a forward masking task. In contrast, other IC neurons, especially onset neurons, respond strongly and reliably to the short probe stimuli and show threshold shifts that are comparable with behavioral ones. This finding is consistent with previous studies of responses to sequential tones in the IC (Finlayson, 1999), and it suggests that the onset neurons may be more heavily weighted by the auditory system for tasks like teasing apart a string of rapidly changing stimuli.

The recovery of response strength with increasing delay between masker and probe (Fig. 7) follows a time course that might be useful for maintaining a robust representation of speciesspecific social communication calls in the marmoset midbrain. One of the most common vocalizations made in this species is the "twitter" call, which is made up of a series of brief frequencymodulated phrases separated by silent intervals ranging from $\sim 100-160$ ms (DiMattina and Wang, 2006). In our data, probe thresholds were recovered in most neurons for silent gaps of this duration. These slow fluctuations would be more likely to be present at the output of IC neurons than faster modulations that might be attenuated by the same mechanisms underlying the forward suppression described here.

\section{Summary}

A forward-masking paradigm modeled after those used in psychoacoustic studies was applied in the awake marmoset IC, revealing level-dependent, frequency tuned offset suppression with characteristics strikingly similar to those of perceptual forward masking. These response profiles were largely emergent between ANFs and IC neurons; indirect evidence points to the de novo generation of many of the key features at the level of the IC, including the excitation-independent, wide dynamic range of masker-evoked shifts in probe detection threshold. 

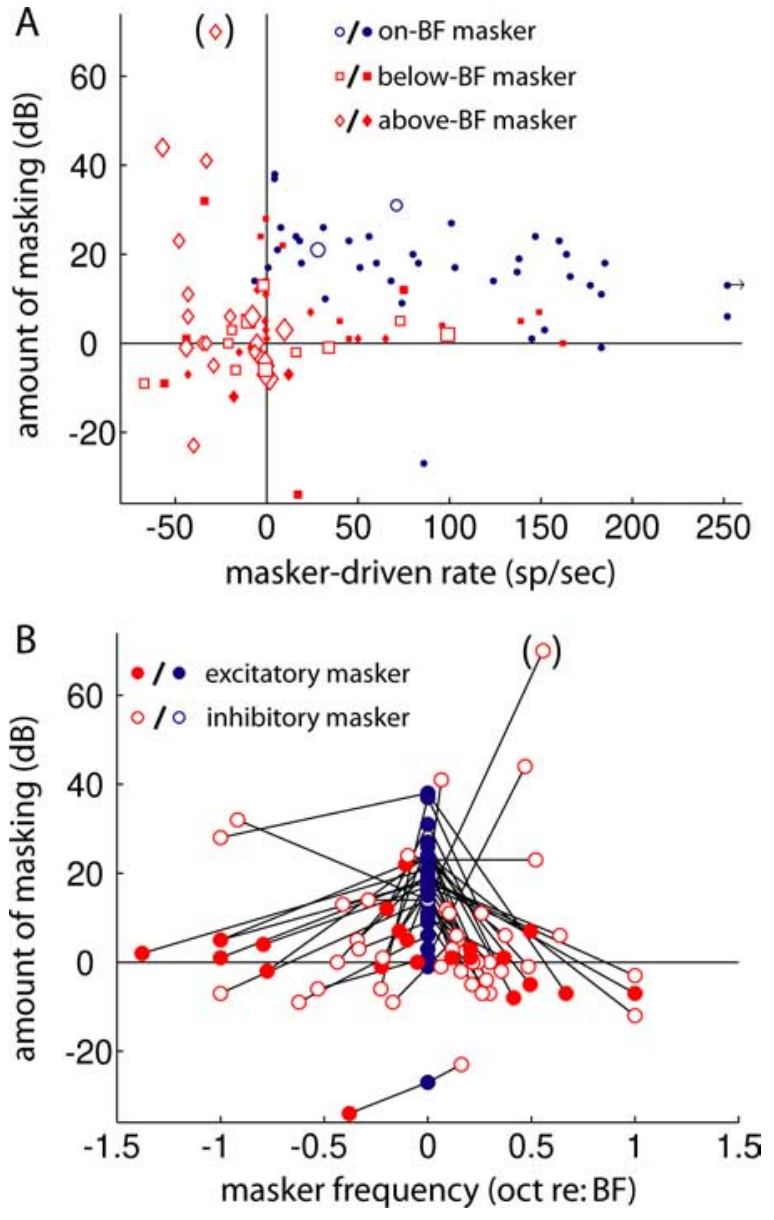

Figure 9. Off-BF tones were often ineffective as forward maskers, despite the fact that they were more likely to elicit a persistent or offset-locked excitatory response. $A$, Amount of masking as a function of masker-driven rate. On-BF (blue circles), below-BF (red squares), and above- $\mathrm{BF}$ (red diamonds) maskers are included; the size of the symbol scales with the $\mathrm{PA}$ ratio for each condition. Rightward arrow in A represents a point with a masker-driven rate of 358 $\mathrm{sp} / \mathrm{s}$. Shaded symbols correspond to a $\mathrm{PA}$ ratio of $<1$. The data points surrounded by parentheses are from a neuron with a probe response that never emerged above the rate caused by the above-BF masker (see Fig. 8Cb). B, Amount of masking (i.e., probe threshold shift) as a function of the masker frequency distance from $B F$ in octaves.

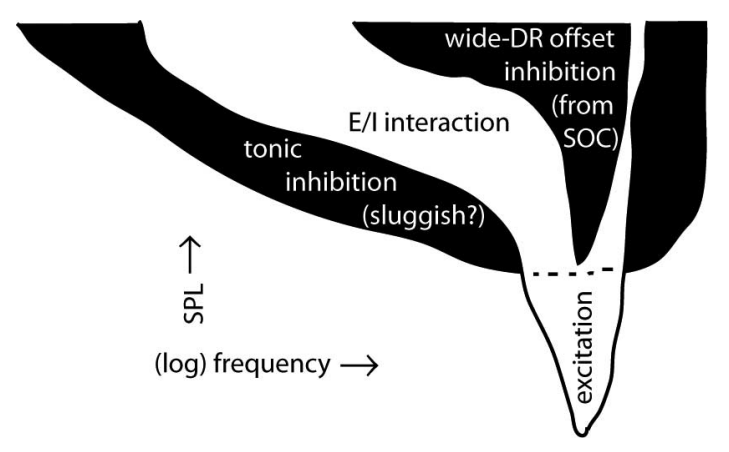

Figure 10. Schematic of the frequency and level dependence of a hypothetical model IC neuron based on features observed in the forward masked responses. DR: dynamic range, SOC: superior olivary complex.

\section{References}

Aitkin LM, Webster WR, Veale JL, Crosby DC (1975) Inferior colliculus. I. Comparison of response properties of neurons in central, pericentral, and external nuclei of adult cat. J Neurophysiol 38:1196-1207.

Backoff PM, Palombi PS, Caspary DM (1997) Glycinergic and GABAergic inputs affect short-term suppression in the cochlear nucleus. Hear Res 110:155-163.

Bartlett EL, Wang X (2005) Long-lasting modulation by stimulus context in primate auditory cortex. J Neurophysiol 94:83-104.

Bartlett EL, Wang X (2007) Neural representations of temporally modulated signals in the auditory thalamus of awake primates. J Neurophysiol 97:1005-1017.

Bauer EE, Klug A, Pollak GD (2000) Features of contralaterally evoked inhibition in the inferior colliculus. Hear Res 141:80-96.

Bleeck S, Sayles M, Ingham NJ, Winter IM (2006) The time course of recovery from suppression and facilitation from single units in the mammalian cochlear nucleus. Hear Res 212:176-184.

Boettcher FA, Salvi RJ, Saunders SS (1990) Recovery from short-term adaptation in single neurons in the cochlear nucleus. Hear Res 48:125-144.

Brosch M, Schreiner CE (1997) Time course of forward masking tuning curves in cat primary auditory cortex. J Neurophysiol 77:923-943.

Brosch M, Schulz A, Scheich H (1999) Processing of sound sequences in macaque auditory cortex: response enhancement. J Neurophysiol 82:1542-1559.

Calford MB, Semple MN (1995) Monaural inhibition in cat auditory cortex. J Neurophysiol 73:1876-1891.

Covey E, Kauer JA, Casseday JH (1996) Whole-cell patch-clamp recording reveals subthreshold sound-evoked postsynaptic currents in the inferior colliculus of awake bats. J Neurosci 16:3009-3018.

Delgutte B (1980) Representation of speech-like sounds in the discharge patterns of auditory-nerve fibers. J Acoust Soc Am 68:843-857.

DiMattina C, Wang X (2006) Virtual vocalization stimuli for investigating neural representations of species-specific vocalizations. J Neurophysiol 95:1244-1262.

Faingold CL, Boersma Anderson CA, Caspary DM (1991) Involvement of GABA in acoustically-evoked inhibition in inferior colliculus neurons. Hear Res 52:201-216.

Faure PA, Fremouw T, Casseday JH, Covey E (2003) Temporal masking reveals properties of sound-evoked inhibition in duration-tuned neurons of the inferior colliculus. J Neurosci 23:3052-3065.

Finlayson PG (1999) Post-stimulatory suppression, facilitation and tuning for delays shape responses of inferior colliculus neurons to sequential pure tones. Hear Res 131:177-194.

Finlayson PG, Adam TJ (1997) Excitatory and inhibitory response adaptation in the superior olive complex affects binaural acoustic processing. Hear Res 103:1-18.

Furukawa S, Maki K, Kashino M, Riquimaroux H (2005) Dependency of the interaural phase difference sensitivities of inferior colliculus neurons on a preceding tone and its implications in neural population coding. J Neurophysiol 93:3313-3326.

Harris DM, Dallos P (1979) Forward masking of auditory nerve fiber responses. J Neurophysiol 42:1083-1107.

Ingham NJ, Hart HC, McAlpine D (2001) Spatial receptive fields of inferior colliculus neurons to auditory apparent motion in free field. J Neurophysiol 85:23-33.

Jesteadt W, Bacon SP, Lehman JR (1982) Forward masking as a function of frequency, masker level, and signal delay. J Acoust Soc Am 71:950-962.

Kelly JB, Liscum A, van Adel B, Ito M (1998) Projections from the superior olive and lateral lemniscus to tonotopic regions of the rat's inferior colliculus. Hear Res 116:43-54.

Kidd G Jr, Feth LL (1982) Effects of masker duration in pure-tone forward masking. J Acoust Soc Am 72:1384-1386.

Kulesza RJ Jr, Berrebi AS (2000) Superior paraolivary nucleus of the rat is a GABAergic nucleus. J Assoc Res Otolaryngol 1:255-269.

Kulesza RJ Jr, Spirou GA, Berebbi AS (2003) Physiological response properties of neurons in the superior paraolivary nucleus of the rat. J Neurophysiol 89:2299-2312.

Kuwada S, Batra R (1999) Coding of sound envelopes by inhibitory rebound in neurons of the superior olivary complex in the unanesthetized rabbit. J Neurosci 19:2273-2287.

Kuwada S, Yin TCT, Haberly LB, Wickesburg RE (1980) Binaural interaction in the cat inferior colliculus: physiology and anatomy. In: Psychophysical, physiological, and behavioral studies in hearing (van den Brink G, Bilsen FA, eds), pp 401-411. Delft, The Netherlands: Delft UP.

Kuwada S, Batra R, Yin TC, Oliver DL, Haberly LB, Stanford TR (1997) Intracellular recordings in response to monaural and binaural stimula- 
tion of neurons in the inferior colliculus of the cat. J Neurosci 17:7565-7581.

LeBeau FE, Malmierca MS, Rees A (2001) Iontophoresis in vivo demonstrates a key role for $\mathrm{GABA}_{\mathrm{A}}$ and glycinergic inhibition in shaping frequency response areas in the inferior colliculus of guinea pig. J Neurosci 21:7303-7312.

Lu T, Liang L, Wang X (2001) Neural representations of temporally asymmetric stimuli in the auditory cortex of awake primates. J Neurophysiol 85:2364-2380.

Lüscher E, Zwislocki J (1949) Adaptation of the ear to sound stimuli. J Acoust Soc Am 21:135-139.

Malone BJ, Semple MN (2001) Effects of auditory stimulus context on the representation of frequency in the gerbil inferior colliculus. J Neurophysiol 86:1113-1130.

McAlpine D, Jiang D, Shackleton TM, Palmer AR (2000) Responses of neurons in the inferior colliculus to dynamic interaural phase cues: evidence for a mechanism of binaural adaptation. J Neurophysiol 83:1356-1365.

Moore BC, Glasberg BR (1983) Growth of forward masking for sinusoidal and noise maskers as a function of signal delay; implications for suppression in noise. J Acoust Soc Am 73:1249-1259.

Moore BC, Glasberg BR, Plack CJ, Biswas AK (1988) The shape of the ear's temporal window. J Acoust Soc Am 83:1102-1116.

Nakamoto KT, Zhang J, Kitzes LM (2006) Temporal nonlinearity during recovery from sequential inhibition by neurons in the cat primary auditory cortex. J Neurophysiol 95:1897-1907.

Nelson PG, Erulkar SD (1963) Synaptic mechanisms of excitation and inhibition in the central auditory pathway. J Neurophysiol 26:908-923.

Oliver DL (2004) Neuronal organization in the inferior colliculus. In: The inferior colliculus (Winer JA, Schreiner CE, eds), pp 69-114. New York: Springer.

Oxenham AJ (2001) Forward masking: adaptation or integration? J Acoust Soc Am 109:732-741.

Oxenham AJ, Plack CJ (2000) Effects of masker frequency and duration in forward masking: further evidence for the influence of peripheral nonlinearity. Hear Res 150:258-266.

Palombi PS, Backoff PM, Caspary DM (1994) Paired tone facilitation in dorsal cochlear nucleus neurons: a short-term potentiation model testable in vivo. Hear Res 75:175-183.

Pedemonte M, Torterolo P, Velluti RA (1997) In vivo intracellular characteristics of inferior colliculus neurons in guinea pigs. Brain Res 759:24-31
Plack CJ, Oxenham AJ (1998) Basilar-membrane nonlinearity and the growth of forward masking. J Acoust Soc Am 103:1598-1608.

Pollak GD, Park TJ (1993) The effects of GABAergic inhibition on monaural response properties of neurons in the mustache bat's inferior colliculus. Hear Res 65:99-117.

Ramachandran R, Davis KA, May BJ (1999) Single-unit responses in the inferior colliculus of decerebrate cats. I. Classification based on frequency response maps. J Neurophysiol 82:152-163.

Relkin EM, Doucet JR (1991) Recovery from prior stimulation. I: relationship to spontaneous firing rates of primary auditory neurons. Hear Res 55:215-222.

Relkin EM, Pelli DG (1987) Probe tone thresholds in the auditory nerve measured by two-interval forced-choice procedures. J Acoust Soc Am 82:1679-1691.

Relkin EM, Turner CW (1988) A reexamination of forward masking in the auditory nerve. J Acoust Soc Am 84:584-591.

Sanes DH, Malone BJ, Semple MN (1998) Role of synaptic inhibition in processing of dynamic binaural level stimuli. J Neurosci 18:794-803.

Scholl B, Gao X, Wehr M (2008) Level dependence of contextual modulation in auditory cortex. J Neurophysiol 99:1616-1627.

Shore SE (1995) Recovery of forward-masked responses in ventral cochlear nucleus neurons. Hear Res 82:31-43.

Smith RL (1977) Short-term adaptation in single auditory nerve fibers: some poststimulatory effects. J Neurophysiol 40:1098-1111.

Starr A (1965) Suppression of single unit activity in cochlear nucleus of the cat following sound stimulation. J Neurophysiol 28:850-862.

Tan ML, Borst JG (2007) Comparison of responses of neurons in the mouse inferior colliculus to current injections, tones of different durations, and sinusoidal amplitude-modulated tones. J Neurophysiol 98:454-466.

Wehr M, Zador AM (2005) Synaptic mechanisms of forward suppression in rat auditory cortex. Neuron 47:437-445.

Widin GP, Viemeister NF (1979) Intensive and temporal effects in puretone forward masking. J Acoust Soc Am 66:388-395.

Xie R, Gittelman JX, Li N, Pollak GD (2008) Whole cell recordings of intrinsic properties and sound-evoked responses from the inferior colliculus. Neuroscience 154:245-256.

Zilany MS, Bruce IC (2007) Representation of the vowel $/ \varepsilon /$ in normal and impaired auditory nerve fibers: model predictions of responses in cats. J Acoust Soc Am 122:402-417. 